\title{
Charge Stripes and Antiferromagnetism in Copper-Oxide
}

\section{Superconductors}

\section{J. M. Tranquada}

Physics Department, Brookhaven National Laboratory, Upton, NY 11973, USA

\section{CONF-971021- -}

RECEIVED

FFR 101998

OSTI

\begin{abstract}
Superconducting cuprate compounds are obtained by doping holes into antiferromagentic insulators. Neutron scattering studies have provided evidence that the doped holes tend to segregate into charge stripes, which act like domain walls between antiferromagnetic regions. The interaction between the spatially segregated holes and the magnetic domains may be responsible for the strong pairing interaction found in the cuprates.
\end{abstract}

KEYWORDS: charge stripes, antiferromagnetism, $\mathrm{La}_{1.6-x} \mathrm{Nd}_{0.4} \mathrm{Sr}_{x} \mathrm{CuO}_{4}$

\section{INTRODUCTION}

The parent compounds of the superconducting cuprates, typified by $\mathrm{La}_{2} \mathrm{CuO}_{4}$, are Mott insulators [1]. That is, they are insulating because the strong onsite Coulomb interaction between the "conduction" electrons causes these electrons to be localized. The relevant electronic states are made up of the $\mathrm{Cu} 3 d_{x^{2}-y^{2}}$ orbitals, and in $\mathrm{La}_{2} \mathrm{CuO}_{4}$ there is precisely one hole per orbital. In second-order perturbation theory the localized holes can lower there energy by making virtual hops to neighboring sites. Because of the Pauli exclusion principle, the hopping can only occur if the hole spins on neighboring sites are antiparallel. Hence, the strong interactions that cause the ground state to be an insulator also lead to antiferromagnetism. It is important to note that the antiferromagnetism of a Mott insulator is quite different from a spin-density-wave state, in which a band gap opens between weakly correlated bands of Bloch states that correspond to spatially-modulated spin densities. A wide range of experimental results on $\mathrm{La}_{2} \mathrm{CuO}_{4}$ overwhelmingly point to a strongly-correlated ground state.

To obtain superconductivity, it is necessary to dope the $\mathrm{CuO}_{2}$ planes with mobile holes, and in $\mathrm{La}_{2} \mathrm{CuO}_{4}$ this can be achieved by partial substitution of $\mathrm{Sr}^{2+}$ for $\mathrm{La}^{3+}$. A very small amount of $\mathrm{Sr}(2 \%)$ is sufficient to kill the antiferromagnetic order [2], and by the time enough $\mathrm{Sr}$ has been added to induce superconductivity ( $>5 \%$ ), any static magnetism has largely disappeared. Dynamic magnetic correlations are still observed, but the characteristic wave vectors of the low-energy magnetic excitations are split incommensurately about the antiferromagnetic point [3]. The incommensurability of the magnetic response led some to an interpretation based on nearly-nested Fermi surface $[4,5,6]$. However, it is now known that the incommensurability grows linearly with $x$ for $x<\frac{1}{8}$ [7], in a manner very similar to that observed in $\mathrm{La}_{2-x} \mathrm{Sr}_{x} \mathrm{NiO}_{4}[8,9]$ (see Fig. 1), a system which remains a correlated insulator even after doping. In the latter case, there is strong evidence from diffraction studies for a coupled ordering of the doped holes and $\mathrm{Ni}$ spins $[8,9,10]$. The

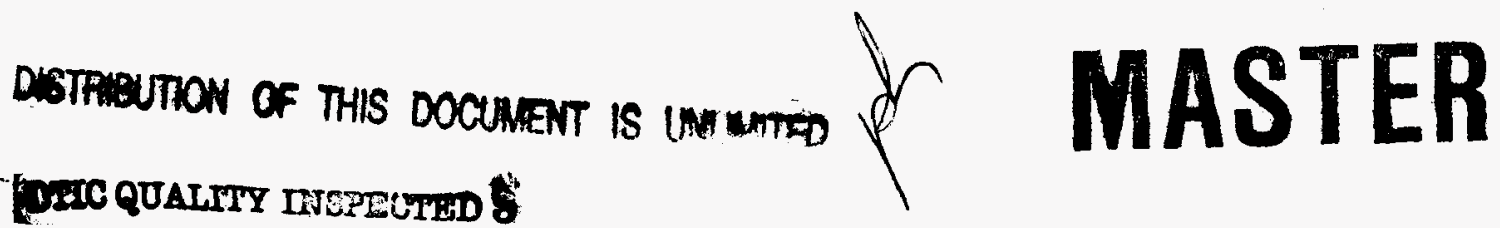



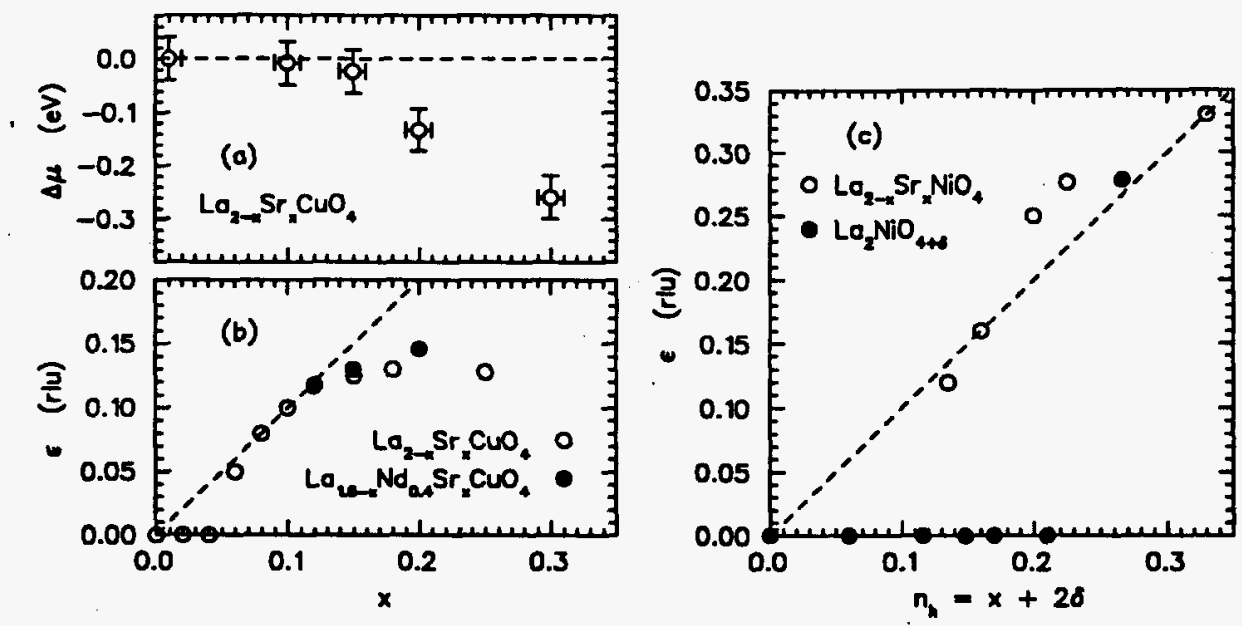

Figure 1: (a) Shift of the chemical potential in $\mathrm{La}_{2} \mathrm{CuO}_{4}$ as a function of $\mathrm{Sr}$ doping, from the photoemission study by Ino et al. [19]. (b) Shift in the incommensurate splitting, $\epsilon$, of the magnetic peaks for $\mathrm{La}_{2-x} \mathrm{Sr}_{x} \mathrm{CuO}_{4}$ (open circles; from inelastic neutron scattering study by Yamada et al. [7]) and $\mathrm{La}_{1.6-x} \mathrm{Nd}_{0.4} \mathrm{Sr}_{x} \mathrm{CuO}_{4}$ (filled circles; from elastic scattering $[20,21])$. (c) Shift in $\epsilon$ for Sr-doped (open circles) and O-doped (filled circles) $\mathrm{La}_{2} \mathrm{NiO}_{4}$ from neutron diffraction (for references, see $[9,22]$ ).

charges order first, in periodically spaced stripes. At a lower temperature, the Ni moments in the intervening regions order antiferromagnetically, with the phase of the magnetic order shifting by $\pi$ on crossing a charge stripe. It has been argued that this charge-stripe order is an example of "topological doping", and that such spatial modulations should be the common response of a correlated insulator to doping [11].

The first theoretical suggestions of charge-stripe correlations in the cuprates came from mean-field analyses of the Hubbard model $[12,13]$. The solution that was found gave insulating stripes with a spacing twice that suggested by experiment [3], and as a result the stripe picture was initially rejected. More recently, however, arguments in favor of dynamical metallic stripe correlations have been presented, based on a range of theoretical approaches $[14,15,16,17,18]$.

\section{STRIPE ORDER IN Nd-DOPED $\mathrm{La}_{2-x} \mathrm{Sr}_{x} \mathrm{CuO}_{4}$}

Direct evidence for charge stripes in the cuprates has been obtained from neutron scattering studies of $\mathrm{La}_{1.6-x} \mathrm{Nd}_{0.4} \mathrm{Sr}_{x} \mathrm{CuO}_{4}$. The isovalent substitution of $\mathrm{Nd}$ for La has no effect on the hole concentration. Its only effect is to induce a subtle change in the lattice structure at low temperatures $(<70 \mathrm{~K})[23,24]$. In contrast to samples without $\mathrm{Nd}$, the superconducting transition temperature $T_{c}$ drops to zero for $x \approx \frac{1}{8}$, and it is finite but significantly depressed at neighboring concentrations. Neutron scattering measurements on a crystal with $x=$ 0.12 revealed superlattice peaks indicating modulated antiferromagnetic order together with a modulated charge density $[20,25]$. The evidence for charge order has recently been confirmed by a high-energy X-ray diffraction study [26]. The charge order clearly occurs at a temperature above that of the magnetic order. Furthermore, muon-spin-rotation measurements indicate that the magnitude of the ordered $\mathrm{Cu}$ moments is approximately 


\section{DISCLAIMER}

This report was prepared as an account of work sponsored by an agency of the United States Government. Neither the United States Government nor any agency thereof, nor any of their employees, makes any warranty, express or implied, or assumes any legal liability or responsibility for the accuracy, completeness, or usefulness of any information, apparatus, product, or process disclosed, or represents that its use would not infringe privately owned rights. Reference herein to any specific commercial product, process, or service by trade name, trademark, manufacturer, or otherwise does not necessarily constitute or imply its endorsement, recommendation, or favoring by the United States Government or any agency thereof. The views and opinions of authors expressed herein do not necessarily state or reflect those of the United States Government or any agency thereof. 
$0.3 \mu_{B}$ [27], half the value found in undoped antiferromagnetic $\mathrm{La}_{2} \mathrm{CuO}_{4}$. These results are consistent with charge-stripe order, where the stripes run parallel to a $\mathrm{Cu}-\mathrm{O}$ bond direction. This orientation of stripes can be pinned by the Nd-induced structural distortion, whereas there would be no coupling to the lattice modulation in the Nd-free structure.

Measurements on crystals with $x=0.15$ and 0.20 demonstrated that elastic incommensurate magnetic peaks are also present at these concentrations, although the order parameter and ordering temperature decrease with increasing $x$ [21]. The incommensurability of the magnetic order is presumably due to charge stripes, although the charge-order scattering is rather difficult to detect. Intriguingly, superconductivity was also observed in these phases $[21,28,29]$. This coexistence demonstrates that stripes and superconductivity are compatible, although the two types of order tend to compete with each other.

If one compares the incommensurate splittings, $\epsilon$, of the magnetic scattering in samples with and without Nd, one finds that they are essentially the same for a given hole concentration as shown in Fig. 1(b). Thus, the correlations in $\mathrm{La}_{2-x} \mathrm{Sr}_{x} \mathrm{CuO}_{4}$ appear to be dynamic versions of the ordered stripes found in the Nd-doped crystals. As mentioned in the introduction, Yamada et al. [7] have found that $\epsilon \approx x$ for $0.05<x<\frac{1}{8}$ in $\mathrm{La}_{2-x} \mathrm{Sr}_{x} \mathrm{CuO}_{4}$. This suggests that, in this range, the hole concentration within the stripes remains fixed (at one hole per two $\mathrm{Cu}$ sites) while the spacing between stripes decreases with increasing hole concentration.

It has been argued by Castellani, Di Castro, and Grilli [30] that the observed charge and spin modulations may be the result of a charge-density-wave instability, rather than local phase separation. In that case, one might expect to see evidence for a significant gap in in-plane charge excitations when charge order occurs; however, the magnitude of the low-temperature resitivity in $\mathrm{La}_{1.48} \mathrm{Nd}_{0.4} \mathrm{Sr}_{0.12} \mathrm{CuO}_{4}$ is inconsistent with a true gap [31], and the infrared reflectivity measurements by Tajima et al. [32] indicate that the in-plane conductivity at $100 \mathrm{~cm}^{-1}$ continues to increase on cooling from $100 \mathrm{~K}$ to $8 \mathrm{~K}$. Recently, Ino et al. [19] have shown through a core-level photoemission study that the chemical potential does not shift over the doping range in which $\epsilon \approx x$ [see Fig. 1(a,b)]. Such behavior is just what one expects for frustrated phase separation in a doped insulator [33].

\section{OTHER CUPRATES AND RELEVANCE TO SUPERCONDUCTIVITY}

It appears now that $\mathrm{Nd}$-doping is not the only way to pin stripes in $\mathrm{La}_{2-x} \mathrm{Sr}_{x} \mathrm{CuO}_{4}$. Hirota et al. [34] have recently shown that substituting $1.2 \% \mathrm{Zn}$ for $\mathrm{Cu}$, with $x=0.14$, induces sharp quasi-elastic peaks corresponding to incommensurate magnetic scattering. There is also new evidence that the magnetic fluctuations in two other superconductors, $\mathrm{La}_{2} \mathrm{CuO}_{4+\delta}$ [35] and $\mathrm{YBa}_{2} \mathrm{Cu}_{3} \mathrm{O}_{6.6}[36]$ are incommensurate. Thus, although further research is needed, it appears that charge-stripe correlations may be a common feature of the underdoped cuprates.

If one accepts that dynamical charge stripes may exist in the cuprates, the next question is: what do they have to do with superconductivity? To start with, it should be clear that a strong spatial modulation of the charge density combined with intervening antiferromagnetic domains is a very unusual situation, and that the pairing mechanism is likely to be novel. One such novel pairing model has been proposed by Emery, Kivelson, 


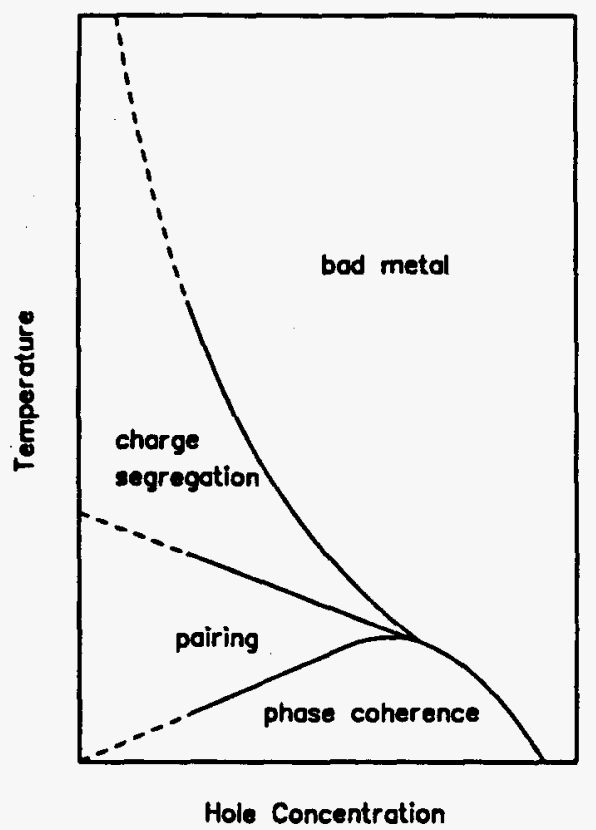

Figure 2: Generic phase diagram for layered cuprates. Details are discussed in the text. (Left out of the diagram is the antiferromagnetically-ordered phase that occurs at very low hole concentrations.

and Zachar [37]. (Some related analysis has been done by Krotov, Lee, and Balatsky [38].) They have considered the simplified case of holes in a one-dimensional metal (charge stripe) interacting with a narrow antiferromagnetic domain. The finite magnetic region of Heisenberg-coupled spins will tend to have a gap in its spin excitations at finite temperatures, and in some geometries (e.g., even-legged ladders) there would be a spin gap even at $T=0$. It is energetically unfavorable for a single hole to move into the magnetic domain; however, a pair of holes can acquire the spin gap by hopping into and out of the domain. Within the charge stripe, the spin gap acts like a pairing amplitude. To develop true superconductivity, it is necessary to develop phase coherence via Josephson coupling between neighboring stripes. The Josephson coupling should increase as the stripes get closer together, so that $T_{c}$ should increase with hole concentration, as observed in underdoped cuprates.

A generic phase diagram for layered cuprate superconductors is shown in Fig. 1. The lowest curve indicates the phase boundary for superconductivity. The upper two lines indicate crossover temperatures that have been identified in a variety of experimental measurements $[39,40]$. One interpretation of the underlying physics is the following. At high temperatures the charge distribution is relatively homogeneous, but the scattering length is quite short. It is not clear that the quasiparticle concept has any meaning in this regime, which has been given the name "bad metal" [41]. Below the upper crossover, the holes begin to segregate into stripes. The spin gap of the antiferromagnetic domains, and the consequent pairing amplitude for holes, begin to manifest themselves below the lower crossover. True superconductivity does not appear until phase coherence is established $[37,40]$. 


\section{CONCLUSION}

There is now significant evidence for stripe correlations coexisting with superconductivity in $\mathrm{La}_{2-x} \mathrm{Sr}_{x} \mathrm{CuO}_{4}$. New experiments indicate that charge stripes may be a generic feature of underdoped cuprate superconductors. A model of hole pairing based on the stripe picture provides an intriguing rationalization for the doping dependence of crossovers and superconducting transition temperatures. Further research is necessary to test these concepts.

\section{ACKNOWLEDGEMENT}

I have benefited from interactions with many experimental collaborators, including J. D. Axe, D. J. Buttrey, G. Shirane, N. Ichikawa, S. Uchida, and P. Wochner. I am also grateful for frequent discussions with V. J. Emery and S. A. Kivelson. This work is supported by Contract No. DE-AC02-76CH00016, Division of Materials Sciences, U.S. Department of Energy.

\section{References}

[1] P. W. Anderson, Adv. Phys. 46, 3 (1997).

[2] R. J. Birgeneau and G. Shirane, in Physical Properties of High Temperature Superconductors, edited by D. M. Ginsberg (World Scientific, Singapore, 1989), pp. 151-211.

[3] S.-W. Cheong et al., Phys. Rev. Lett. 67, 1791 (1991).

[4] J. P. Lu, Q. Si, J. H. Kim, and K. Levin, Phys. Rev. Lett. 65, 2466 (1990).

[5] P. B. Littlewood, J. Zaanen, G. Aeppli, and H. Monien, Phys. Rev. B 48, 487 (1993).

[6] T. Tanamoto, H. Kohno, and H. Fukuyama, J. Phys. Soc. Jpn. 63, 2739 (1994).

[7] K. Yamada et al., Proc. of $\mathrm{M}^{2} \mathrm{~S}-\mathrm{HTSC}$, to be published in Physica C.

[8] C. H. Chen, S.-W. Cheong, and A. S. Cooper, Phys. Rev. Lett. 71, 2461 (1993).

[9] J. M. Tranquada, D. J. Buttrey, and V. Sachan, Phys. Rev. B 54, 12318 (1996).

[10] J. M. Tranquada, D. J. Buttrey, V. Sachan, and J. E. Lorenzo, Phys. Rev. Lett. 73, 1003 (1994).

[11] S. A. Kivelson and V. J. Emery, Syn. Met. 80, 151 (1996).

[12] J. Zaanen and O. Gunnarsson, Phys. Rev. B 40, 7391 (1989).

[13] H. J. Schulz, J. Phys. (Paris) 50, 2833 (1989).

[14] U. Löw, V. J. Emery, K. Fabricius, and S. A. Kivelson, Phys. Rev. Lett. 72, 1918 (1994).

[15] L. Chayes et al., Physica A 225, 129 (1996).

[16] C. Nayak and F. Wilczek, Phys. Rev. Lett. 78, 2465 (1997).

[17] S. R. White and D. J. Scalapino, Report No. cond-mat/9705128. 
[18] A. H. Castro Neto, Z. Phys. B 103, 185 (1997).

[19] A. Ino et al., Phys. Rev. Lett. 79, 2101 (1997).

[20] J. M. Tranquada et al., Nature 375, 561 (1995).

[21] J. M. Tranquada et al., Phys. Rev. Lett. 78, 338 (1997).

[22] S.-H. Lee and S.-W. Cheong, Phys. Rev. Lett. 79, 2514 (1997).

[23] M. K. Crawford et al., Phys. Rev. B 44, 7749 (1991).

[24] B. Büchner, M. Breuer, A. Freimuth, and A. P. Kampf, Phys. Rev. Lett. 73, 1841 (1994).

[25] J. M. Tranquada et al., Phys. Rev. B 54, 7489 (1996).

[26] M. von Zimmermann et al., (preprint).

[27] B. Nachumi and Y. J. Uemura, (private communication).

[28] J. E. Ostenson et al., Phys. Rev. B 56, 2820 (1997).

[29] A. R. Moodenbaugh, L. H. Lewis, and S. Soman, Physica C (in press).

[30] C. Castellani, C. D. Castro, and M. Grilli, Z. Phys. B 103, 137 (1997).

[31] Y. Nakamura and S. Uchida, Phys. Rev. B 46, 5841 (1992).

[32] S. Tajima et al., in Proc. of SNS'97, to be published in J. Phys. Chem. Solids.

[33] V. J. Emery and S. A. Kivelson, Physica C 209, 597 (1993).

[34] K. Hirota, K. Yamada, I. Tanaka, and H. Kojima, (preprint).

[35] B. O. Wells et al., Science 277, 1067 (1997).

[36] P. Dai, H. A. Mook, and F. Doğan, Report No. cond-mat/9707112.

[37] V. J. Emery, S. A. Kivelson, and O. Zachar, Phys: Rev. B 56, 6120 (1997).

[38] Y. A. Krotov, D.-H. Lee, and A. V. Balatsky, Phys. Rev. B 56, 8367 (1997).

[39] B. Batlogg and V. J. Emery, Nature 382, 20 (1996).

[40] V. J. Emery and S. A. Kivelson, in Proc. of SNS'97, to be published in J. Phys. Chem. Solids.

[41] V. J. Emery and S. A. Kivelson, Phys. Rev. Lett. 74, 3253 (1995). 
M98002641

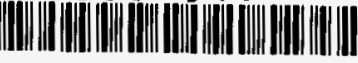

Report Number (14) $3 N C-65025$
CONF-971021-

دubl. Date (11) 199801

ìponsor Code (18) DOE LER,XF

JC Category (19) UE- $U 10, D O E / E R$

DOE 